\title{
A Hybridized Robust Watermarking Scheme based on Fast Walsh-Hadamard Transform and Singular Value Decomposition using Genetic Algorithm
}

\author{
K.Meenakshi \\ $\mathrm{PhD}$ Scholar \\ Department of E.C.E, JNTU \\ Kakinada, Andhra Pradesh
}

\author{
Ch.Srinivasa Rao \\ Professor \\ Department of E.C.E, JNTU college of Engineering \\ Vizianagaram, Andhra Pradesh
}

\author{
K.Satya Prasad \\ Professor \\ Department of E.C.E, JNTU \\ Kakinada, Andhra Pradesh
}

\begin{abstract}
In this paper a robust Hybridized Watermarking scheme based on Fast Walsh Hadamard transform (FWHT) and Singular Value Decomposition (SVD) using Genetic algorithm (GA) is presented. The host image is subjected to FWHT and SVD . The singular values of SVD of host image are modified with singular values of watermark. Multiple scaling factors are used in watermark embedding. The GA searches for optimized multiple scaling factors from a random population using a fitness function. When the optimized multiple scaling factors are applied dramatically lot of improvement is seen in robustness without losing the transparency.
\end{abstract}

\section{General Terms:}

Image processing, Watermarking, Network Security

\section{Keywords:}

Genetic Algorithm, Walsh-Hadamard Transform, Singular Value Decomposition, Multiple Scaling factors, Fitness function

\section{INTRODUCTION}

In recent years the tremendous growth in digital technology and wide spread use of high speed Internet has facilitated the ease with which the multimedia files such as image, audio and video are pirated and reproduced[23]. Consequently, the owners of multimedia document are facing with the problem of protecting their digital property against copyright violation, illegal distribution and unauthorized tampering . Therefore Digital Watermarking emerged as a potential solution to protect multimedia documents from illegal and malicious copying . Digital watermarking is a method to hide some auxiliary information that is integrated with a multimedia object[29]. The watermark is usually a pseudo random number sequence, copyright messages and ownership logo. The two common methods used in watermarking are spatial and spectral domain.Algorithms in first category embed a watermark by modifying the pixel values of image and algorithms in the second category embed watermark in spectrum coefficients after the image has been transformed to spectrum domain such as Discrete Cosine Transform (DCT) [17, 7, 16, 24] , Singular Value Decomposition(SVD) [18, 31 4, 5] Discrete Fourier Transform (DFT) [27, 34, 19] 8], and
Discrete Wavelet Domain(DWT)[12],Complex Hadamard transform [9], Walsh-Hadamard transform [10 11]. The watermark is embedded in spectral coefficients and later inverse transform is used to get watermarked image. The spectral domain techniques have several advantages over spatial domain. First,in spectral domain, most of the energy is packed in few transform coefficients. So watermark can be robustly embedded in few significant transform coefficients.Second, because of Multiresolution properties of spectral domain, watermark can be redistributed over different bands of spectrum coefficients. So spectral based watermarking schemes will improve the transparency of the watermark. Third, the watermark is distributed randomly at different frequency bands, so it become difficult for the hacker to remove the watermark.[22]. Fourth, it is robust against signal processing and compressional attacks. Fifth, some transforms like DFT is robust to affine transforms. Other than these two techniques, to achieve a balance between imperceptibility and robustness, scaling factor of watermark is tuned according to the properties of Human Visual system (HVS), so the distortions presented by watermark are under just noticeable difference [15] . Among the transforms, DCT and DWT are frequently used because they are widely exploited in compression standards. Other than these techniques, watermarking schemes are developed for optimization of imperceptibility and robustness simultaneously as these two parameters are found to be mutually conflicting. So optimization and soft computing tools such as Genetic Algorithm, Bacterial Foraging, Fuzzy logic and Particle Swarm Optimization techniques are used [2].Soft-Computing, a sub-branch of computer science is rich with many optimization tools[20]. In Digital Watermarking,GA may be used to design several optimized algorithms for better trade-off in imperceptibility, robustness and security . In Ref.[30] selected a fitness function derived based on Peak Signal to Noise ratio(P.S.N.R) computed between host and watermarked image and Normalized Cross Correlation(N.C.C) between original and extracted watermark under different attacks. In that paper the host image after DCT transformation segmented into $8 \times 8$ blocks and four bands of size $8 \times 8$ with best fitness value are selected for watermark embedding. It is found that the watermarked scheme proposed by [30] results improved P.S.N.R and N.C.C. In Ref.[5] an optimal watermarking scheme developed based on S.V.D. and GA.The singular values of original image were modified by multiple scaling factors to embed the watermark.They proved the superiority of using multiple scaling factors over single scaling factor 
(SF)in achieving more robustness to attacks. Besides [14] utilized the GA in an embedded technique that was based on Quantization Index Modulation (QIM) technique. In Ref. [33] amelioration of Peak Signal to noise ratio and Bit Correct Rate is obtained using a watermarking scheme based on parametric Slant Transform and GA.

\section{PRELIMINARIES}

This paper is a research work on robust hybrid watermarking based on Fast Walsh-Hadamard Transform and SVD using Genetic Algorithm. A Preliminary introduction of WHT, SVD and GA is given in subsection. 2.1, 2.2 and .2.3

\subsection{Walsh-Hadamard transform}

Walsh-Hadamard transform [25]is a square array of plus and minus ones whose rows and columns are orthogonal to one another. The transform has the advantage of [13] less computational complexity and nearly identical forward and inverse kernels and it is computed by a fast successive doubling method. The algorithm shows robustness against compression at low quality factor 26. The orthogonal rows and columns in Walsh transform are independent. So it is robust for image modification than other popular transforms because in latter the values of pixels in block are changed by different amount due to the multi valued kernels. So it is better suited for real time implementation of watermarking in hardware.It is the lowest computational transform among all the existing orthogonal transforms and is derived from functionally identical kernels. Forward Walsh-Hadamard transform kernel is given in [13]

$$
g(x, y, u, v)=\frac{1}{N}(-1) \sum_{i=0}^{n-1}\left[b_{i}(x) b_{i}(u)+b_{i}(y) b_{i}(v)\right]
$$

Inverse Walsh-Hadamard transform kernel is given in [13

$$
h(x, y, u, v)=\frac{1}{N}(-1) \sum_{i=0}^{n-1}\left[b_{i}(x) b_{i}(u)+b_{i}(y) b_{i}(v)\right]
$$

where $b_{k}(z)$ is the $k^{t h}$ bit of $\mathrm{z}$ in binary representation. The sequency is the number of zero crossings or sign changes in rows or columns of kernel of Walsh-Hadamard transform is analogous to frequency in Fourier transform .

The forward 2D Walsh-Hadamard transform is given by

$$
H(u, v)=\frac{1}{N} \sum_{x=0}^{N-1} \sum_{y=0}^{N-1} f(x, y)(-1) \sum_{i=0}^{n-1}\left[b_{i}(x) b_{i}(u)+b_{i}(y) b_{i}(v)\right]
$$

for $\mathrm{u}, \mathrm{v}=0,1,2, \cdots, \mathrm{N}-1$. The inverse 2D Walsh-Hadamard transform is given by

$$
f(x, y)=\frac{1}{N} \sum_{u=0}^{N-1} \sum_{v=0}^{N-1} H(u, v)(-1) \sum_{i=0}^{n-1}\left[b_{i}(x) b_{i}(u)+b_{i}(y) b_{i}(v)\right]
$$

for $\mathrm{x}, \mathrm{y}=0,1,2, \cdots, \mathrm{N}-1$.

The Hadamard based watermarking schemes already found in literature.In Ref. [6]the watermarking is done based on Multiresolution Walsh-Hadamard transform and SVD. The authors point that as LL band is resistant to attacks and $\mathrm{HH}$ band preserves transparency so middle singular values are chosen for watermark embedding to prevent the hacker to decrypt the watermark.They used the middle singular values of $\mathrm{HH}$ band to embed the watermark. The technique is imperceptible and robust to number of attacks. In Ref.[28] a Hadamard transform based watermarking scheme is used for visible and invisible watermarking.The scaling factors chosen based on sigmoid function. The two algorithms are compared with the proposed one in Section.5

\subsection{Singular Value Decomposition}

SVD is a matrix factorization technique widely used in image compression, retrieval and watermarking. The transform can be applied to square and rectangular matrices of real and complex values. According to it, every real matrix A can be decomposed into a product of three matrices, $\mathrm{U}, \mathrm{S}, \mathrm{V}$ where $U U^{T}=I, V V^{T}=I$ and $\mathrm{S}$ is a diagonal matrix containing largest singular values. The singular values above rank $\mathrm{r}$ of matrix is zero.

$$
A=U S V^{T}
$$

In SVD singular values represent the strong edges and singular vectors represent weak edges and texture region.e[3]. The main advantage of the SVD is the largest singular values packs most of the energy and are robust to small modifications and there by immune to most of the signal processing and image compression operations [21].

In watermarking, the watermark is scaled by a scaling factor and added to host image for obtaining watermarked image. When the scaling factor is large the transparency suffers and the robustness is improved. Contrary is true if the scaling factor is made smaller.Different transform coefficients may exhibit different tolerances to the modification, So single scaling factor is inefficient.So multiple scaling factors are used in watermark embedding and extraction to reduce visible artifacts [5].

\subsection{Genetic Algorithm}

Genetic Algorithm is a heuristic method to obtain useful solutions by trial and error. GA search for a solution to optimization problems using techniques mimicking natural biological evolution, such as hereditary, mutation, selection and cross over. The GA then evaluates each candidate according to the fitness function.Finally GA tries to maximize or minimize the value obtained by fitness function. The GA is initiated by generating random population. Suppose the solution is of size $n$ variables. A population $M$ of size $n$ variables is taken as initial population and each variable is applied to fitness function. In $\mathrm{M}$ iterations the whole population is evaluated.Later out of $\mathrm{M}$, the strongest fitness individuals are stochastically selected and become parents to next generation. After the selection, GA applies operators such as cross over and mutation on the selected parents to alter the features of succeeding generations. The algorithm terminates when the algorithm produces maximum number of generations, or a satisfactory fitness level has been reached for the population. The main components used in Genetic algorithm are

(1) Selection: It is based on Darwinian principle of survival of the fittest. According to it, only the strong population survive in the next generation and weak ones are perished. Similarly in selection process, low fitness individuals are discarded and out of population of size $\mathrm{N}, N_{\text {good }}$ individuals survive for mating and the left over $N-N_{\text {good }}$ are discarded to make room for offspring in the next generation.

(2) Cross over: In cross over two parents are selected from $N_{\text {good }}$ individuals to produce new offspring. A cross over point is selected between the first and last element of chromosomes of parents. Then from cross over point the tails of parents are exchanged to produce new offspring. 
(3) Mutation: It introduces new traits in the offspring completely different from their predecessors. So mutation introduces genetic diversity in population.

\section{WATERMARK EMBEDDING ALGORITHM USING FWHT2D AND SVD}

The watermarking scheme used a hybridization of FWHT2D and SVD. The algorithmic steps for watermark embedding is shown in Figure 1

\subsection{Watermark Embedding}

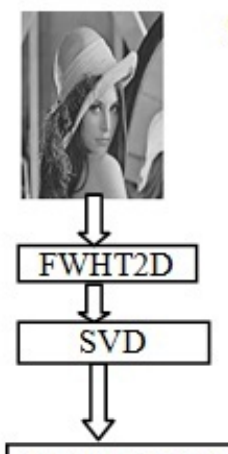

Original image
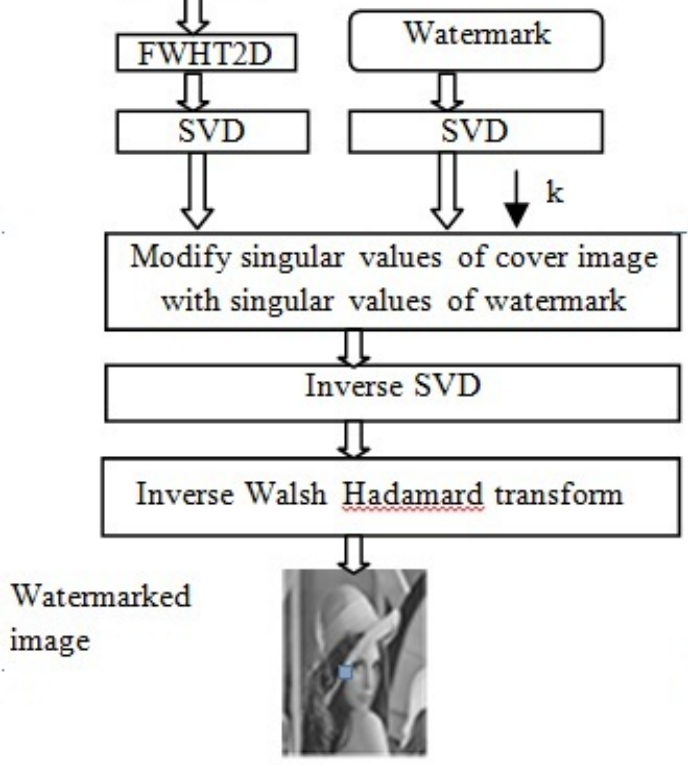

Fig. 1. Embedding Algorithm using FWHT2D and SVD

(1) Let $\mathrm{A}$ be the host image of size $\mathrm{N} \times \mathrm{N}$. Apply Fast 2D Walsh transform to the whole cover image as given in [6]

$$
B=F W H T 2 D(A)
$$

(2) Apply SVD to the Walsh-Hadamard transformed Image

$$
\left[U_{c}, S_{c}, V_{c}\right]=S V D(B)
$$

(3) Apply SVD to the watermark Image W.

$$
\left[U_{w}, S_{w}, V_{w}\right]=S V D(W)
$$

(4) Singular values of watermarked image is obtained by singular values of $S_{c}$ and singular values of watermark $S_{w}$ using the equation below

$$
S_{w a}=S_{c}+\alpha \times S_{w} \times \frac{K}{K_{\max }}
$$

where $K_{\max }$ is the largest coefficient in multiple scaling factors and $\alpha$ is set equal to 0.001 .

(5) Apply inverse SVD

$$
B_{\text {new }}=U_{c} S_{w a} V_{c}^{T}
$$

(6) Apply inverse Walsh-Hadamard transform to obtain the watermarked image.

$$
A_{W}=I F W H T 2 D\left(B_{\text {new }}\right)
$$

\subsection{Watermark Extraction Algorithm using FWHT2D and SVD}

Watermark is extracted using the algorithm given in Figure 2 The proposed algorithm is non-blind and requires host image for extraction.

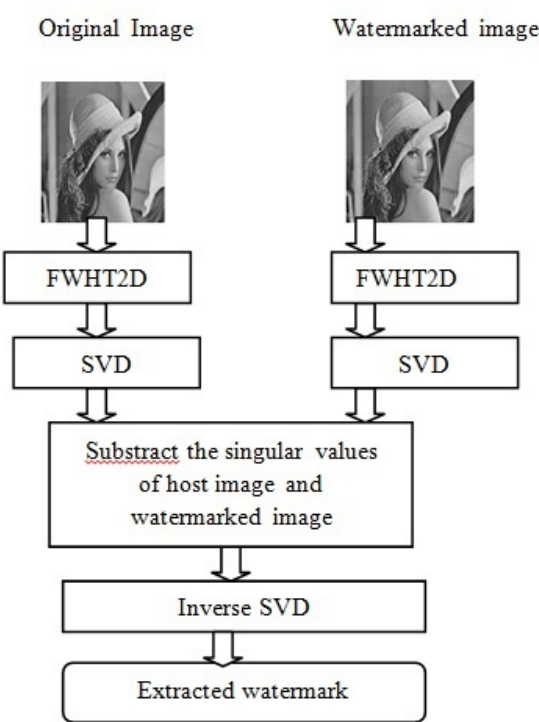

Fig. 2. Extraction Algorithm using FWHT2D and SVD

(1) The multiple scaling factor used in embedding is also utilized in extraction.

(2) Apply Fast Walsh-Hadamard transform to Watermarked image denoted by $A^{\prime}$.

$$
B^{\prime}=F W H T 2 D\left(A^{\prime}\right)
$$

(3) Apply SVD to the Walsh-Hadamard transformed watermarked image.

$$
\left[U_{w a}, S_{w a}, V_{w a}\right]=S V D\left(B^{\prime}\right)
$$

(4) Obtain singular values of watermark from singular values of watermarked image from singular values of original image.

$$
S_{w n e w}=\left(S_{w a}-S_{c}\right) \frac{k_{\max }}{k \times \alpha}
$$

(5) Apply inverse SVD to obtain extracted watermark.

$$
W_{\text {ext }}=U_{w} S_{w n e w} V_{w}^{T}
$$

The proposed watermarking scheme presents high peak signal to noise ratio but it is not withstanding to any type of attack as shown in Table. 1 


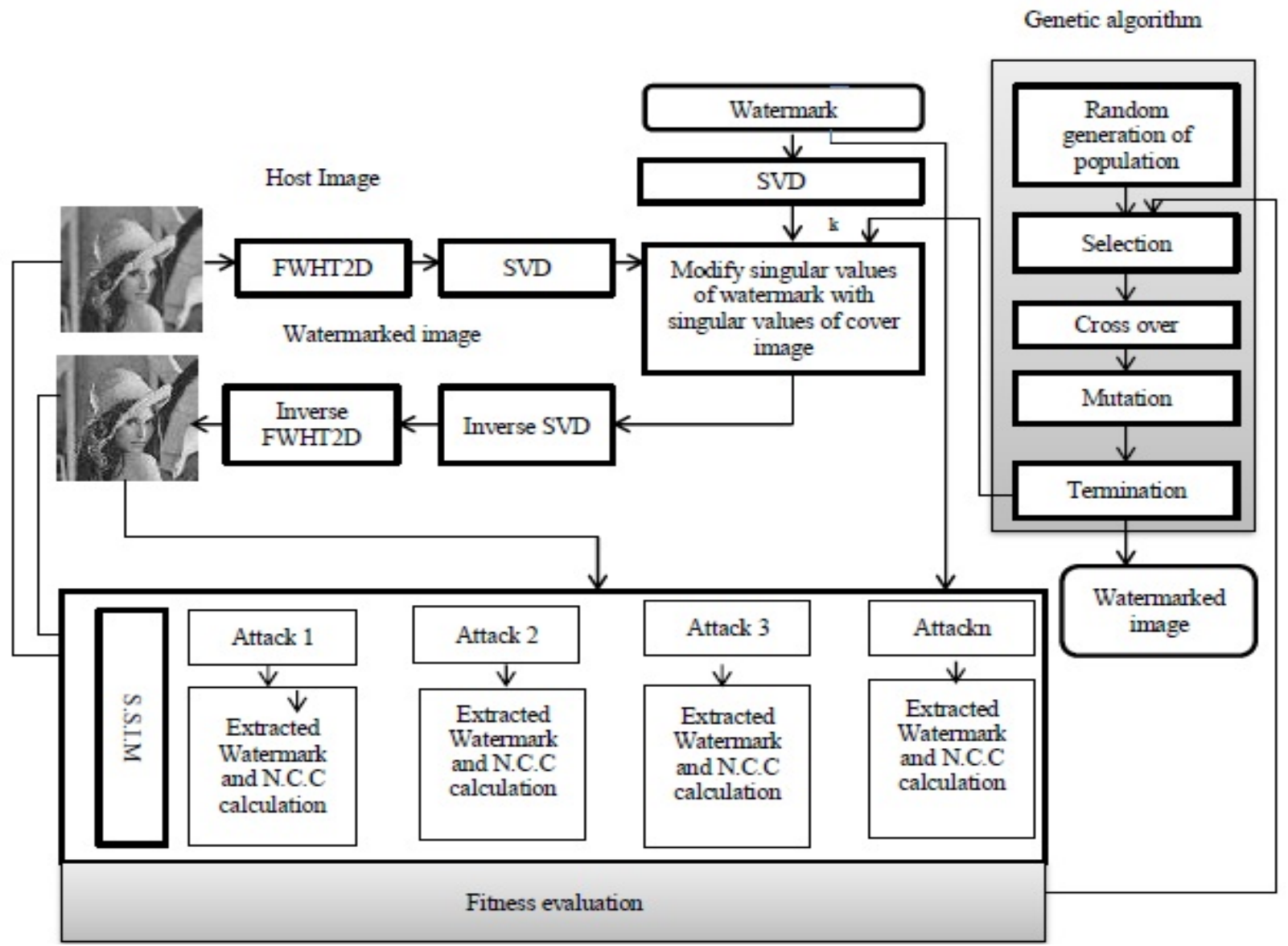

Fig. 3. Block diagram of FWHT2D-SVD based GA

\section{WATERMARK EMBEDDING IN HYBRIDIZED FWHT2D-SVD WITH GA}

The proposed scheme uses hybridized FWHT2D-SVD with GA .The steps used for computation of multiple scaling factors is given below:

(1) As the watermark size is $64 \times 64$, the size of scaling factor to be optimized is taken 64 . The 64 values of scaling factor is taken as chromosome.

(2) In GA, each chromosome in the population represents a possible solution. The strings are enciphered using a double vector.

(3) For initial population, the values of scaling factors(SF)s are randomly produced. If the size of population is $\mathrm{M}$ and the number of variables used is $\mathrm{n}$, then the initial population consists of $M \times n$. So it is a matrix of random values of $M \times n$. In this paper $\mathrm{M}=100$ and $\mathrm{n}=64$ is used.

(4) The watermarks are extracted from the distorted using the extraction procedure discussed earlier. The fitness function is computed based on Structural similarity (S.S.I.M) between original and watermarked image as given in Equ. 18 and Normalized cross correlation between original and extracted wa- termarks as given in Equ. ??

$$
f_{i}=\frac{\sum_{i=1}^{t}\left(N C C\left(w, w_{e x t}\right)\right.}{t}-\operatorname{S.S.I.M~}\left(I, I_{w}\right)
$$

where $\mathrm{t}$ is number of attacks employed. $w$ is the watermark used in embedding. $w_{\text {ext }}$ is the retrieved watermark under each attack. $I$ is the host image $I_{w}$ is the distorted or watermarked image. In order to calculate fitness value, the expression is given as

$$
\text { fitness }_{i}=\frac{1}{f_{i}}
$$

Having evaluated all chromosomes in a generation, the chromosomes that produced the maximum fitness values are selected for the next generation. The population of the next generation is then produced from these chromosomes by using genetic operators such as crossover and mutation operators. The processes explained above are iterated until a termination criteria is met, for example the maximum number of generations.

(5) Evaluate the fitness of each chromosome by passing it to objective function.

(6) Apply selection operator for selecting parents for cross-over There are several selection operators can be used such as 
Roulette-wheel, Boltzmann, tournament, Rank and steadystate selection.

(7) Apply crossover operator for producing population enriched with better individuals. The cross over operators can be used in GA are single point, two-point, multi-point and uniform cross over.

(8) Apply operator mutation such as flipping bits or by using shift left or right operators.

(9) Define cross over rate that define the number of pairs to be cross over for a fixed population.

(10) Define mutation rate that define the number of bits to be mutated.

(11) Define elitism, the population that is retained in next generation.

(12) repeat the steps till termination criteria that is the population have attained a degree of homogeneity.
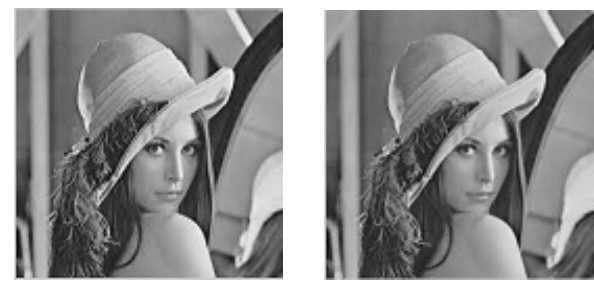

Fig. 4. a) Original image b) Watermarked image.

The Structural Similarity Index is defined in [32]

$$
\operatorname{SSIM}(x, y)=\frac{\left(2 \mu_{x} \mu_{y}+C_{1}\right)+\left(2 \sigma_{x y}+C_{2}\right)}{\left(\mu_{x}^{2}+\mu_{y}^{2}+C_{1}\right)+\left(\sigma_{x}^{2}+\sigma_{y}^{2}+C_{2}\right)}
$$

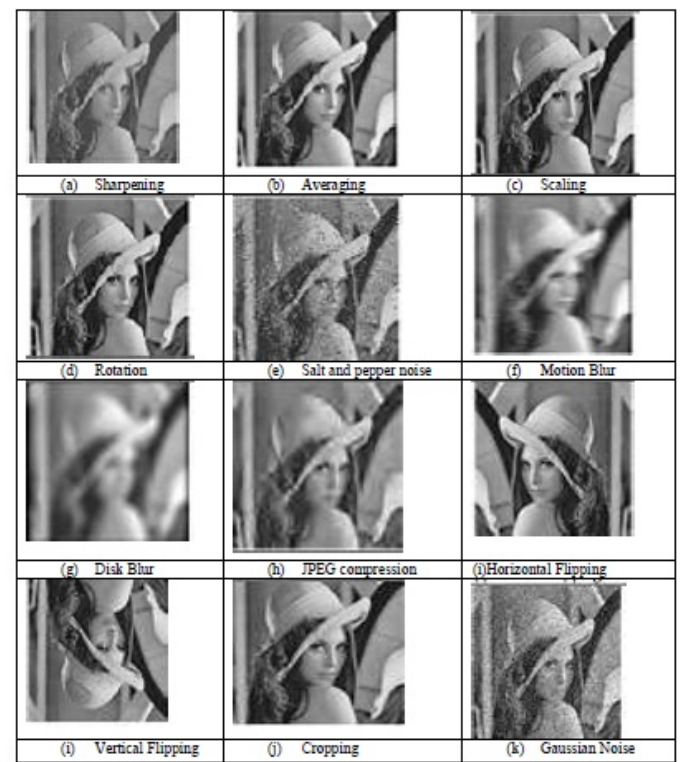

Fig. 5. Signal processing and compressional attacks applied to Lena

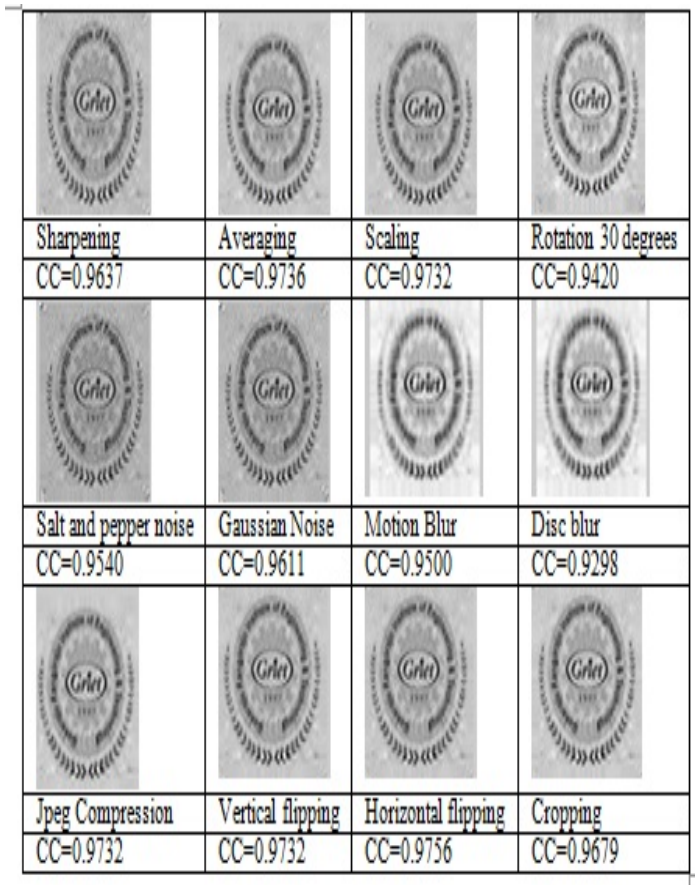

Fig. 6. Extracted watermarks after Lena image is subjected to Signal processing and Compressional attacks

Table 1. Normalized Cross Correlation between original and extracted watermark for different attacks without and with GA

\begin{tabular}{ccc}
\hline \hline Attack type & N.C.C without GA & N.C.C with GA \\
\hline \hline Sharpening & 0.1186 & 0.9637 \\
Averaging & 0.1195 & 0.9736 \\
Scaling & 0.1192 & 0.9732 \\
Rotation & 0.1097 & 0.9420 \\
Salt and Pepper noise & 0.1194 & 0.9540 \\
Gaussian noise & 0.1186 & 0.9611 \\
Motion Blur & 0.1209 & 0.9500 \\
Disk Blur & 0.1215 & 0.9288 \\
Horizontal flip & 0.1191 & 0.9732 \\
Vertical flip & 0.1191 & 0.9732 \\
Cropping & 0.1177 & 0.9756 \\
Jpeg Compression & 0.1192 & 0.9679 \\
\hline
\end{tabular}

where

$\mu_{x}-$ Averageof $x$

$\mu_{y}-$ Averageofy

$\sigma_{x}^{2}$-Variance of $\mathrm{x}$.

$\sigma_{y}^{2}$-Variance of $\mathrm{y}$.

$\sigma_{x y}$-CoVariance of xy.

$C_{1}=K_{1} L^{2}$-constant to avoid instability when $\mu_{x}^{2}+\mu_{y}^{2}$ is close to zero. $k_{1}=0.01, \mathrm{~L}=255$.

$C_{2}=K_{2} L^{2}$-constant to avoid instability when $\sigma_{x}^{2}+\sigma_{y}^{2}$ is close to zero. $k_{2}=0.01, \mathrm{~L}=255$. The fitness value is computed based on Structural Similarity Index measure between original and watermarked image and N.C.C between original and extracted watermark under 12 different attacks.A randomly generated scaling fac- 
Table 2. P.S.N.R and Cross correlation obtained with GA for test images under no attack

\begin{tabular}{ccc}
\hline \hline Test Image & P.S.N.R & C.C \\
\hline Lena & 54.776 & 0.99 \\
F.16 & 55.676 & 1 \\
House & 53.55 & 1 \\
Boat & 53.25 & 1 \\
\hline
\end{tabular}

tor is used in proposed watermarking scheme without GA and from Table 1 low values of cross correlation reveal that the WalshHadamard transform-SVD are not suited for robust watermarking. But with the multiple scaling factor obtained from GA using the fitness function given in Equ. 16. s applied there is dramatic improvement is seen in robustness.

\section{RESULTS AND DISCUSSION}

The numerical simulation of our algorithm is implemented using an Intel 5 processor of $2.5 \mathrm{GHz}$ and 4 GB RAM using Microsoft Windows 7 and Matlab 8.1. We used the $512 \times 512$ gray scale Lena image , $64 \times 64$ gray scale GRIET emblem as host image and logo. The choice of attacks used for evaluating the proposed watermarking scheme are sharpening, averaging, rotation, scaling, salt and pepper noise, Gaussian noise, motion blur, disk blur, Jpeg compression, vertical flipping, horizontal flipping and cropping as shown in Figure 5 The extracted watermarks under different attacks are shown in Figure 6 The watermarking scheme is evaluated through Peak Signal to Noise Ratio (PSNR), [1], Normalized Correlation Coefficient (NCC) [1]. Normally, in image processing, the quality of watermark is judged based on P.S.N.R. The P.S.N.R between watermarked and the original image is defined in equ 19

$$
P S N R=10 \log _{10}\left(\frac{255^{2}}{M . S . E}\right)
$$

Where and the MSE is the mean squared error between host and watermarked image. and Normalized cross correlation is taken as

$$
N . C . C=\frac{\sum_{i=0}^{M-1} \sum_{j=0}^{N-1} w(i, j) w_{e x t}(i, j)}{\sum_{i=0}^{M-1} \sum_{j=0}^{N-1} w(i, j)^{2}}
$$

N.C.C is a measure of similarity between the original and extracted watermark. It lies between 0 to 1 . If it is nearly 1 then the original and extracted watermark are identical.The more the normalized cross correlation the robust the design is.The GA is initialized with different initial population.For each initial population the range of scaling factor must be given. It is a diagonal matrix with 64 variables. The size of population is 100 . So a random matrix comprised of $64 \times 100$ is the initial population.Each 64 variables constitute a chromosome.So initially 100 chromosomes are taken and fed to fitness function and 100 fitness values are obtained. Out of them, the chromosomes having the highest fitness value are retained using selection process. Roulette selection process is used in proposed algorithm. The scaling function used is Rank. The cross over method used is single point. The cross over fraction and mutation rate is taken as 0.8 and 0.04 . The elite count is taken as 5.The number of Generations used is 400 .

The 64 best scaling factor obtained with GA.

328.5014951 .6928655 .8299729 .8926694 .2096689 .4976 605.0334375 .9066764 .0373603 .0104365 .1091959 .9923
Table 3. Comparision of Normalized cross correlation between original and extracted watermark for different attacks between proposed algorithm and santhi et. al

\begin{tabular}{ccc}
\hline \hline Attack type & N.C.C of [28] & N.C.C of proposed Algorithm \\
\hline \hline Scaling & 0.8441 & 0.9732 \\
Rotation & 0.8793 & 0.9420 \\
Salt and Pepper noise & & 0.9540 \\
Gaussian noise & & 0.9611 \\
Motion Blur & & 0.9500 \\
Disk Blur & & 0.9288 \\
Horizontal flip & & 0.9732 \\
Vertical flip & 0.8562 & 0.9732 \\
Cropping & 0.8436 & 0.9756 \\
Jpeg Compression & & 0.9679 \\
\hline
\end{tabular}

Table 4. Comparision of Normalized cross correlation between original and extracted watermark for different attacks between proposed algorithm and bhatnagar et al.

\begin{tabular}{ccc}
\hline \hline Attack type & N.C.C of [6] & N.C.C of proposed Algorithm \\
\hline \hline Sharpening & 0.8890 & 0.9637 \\
Averaging & 0.6690 & 0.9736 \\
Scaling & 0.4822 & 0.9732 \\
Rotation & 0.8289 & 0.9420 \\
Salt and Pepper noise & 0.545 & 0.9540 \\
Gaussian noise & 0.612 & 0.9611 \\
Motion Blur & 0.4822 & 0.9500 \\
Disk Blur & 0.5322 & 0.9288 \\
Horizontal flip & 0.1191 & 0.9732 \\
Vertical flip & 0.1191 & 0.9732 \\
Cropping & 0.8473 & 0.9756 \\
Jpeg Compression & 0.894 & 0.9679 \\
\hline
\end{tabular}

934.2089858 .6199653 .9487962 .2642840 .3048957 .1775 840.3048957 .1775683 .5209601 .4993964 .7182558 .1196 803.6664804 .3353668 .7058540 .7351745 .5223655 .3275 799.7824505 .8513424 .9317875 .3336696 .5437860 .1560 411.3766679 .1339708 .9430716 .8215900 .8159948 .6748 723.8566493 .8747793 .2786762 .7139881 .4497857 .5746 319.9588703 .5539770 .0906871 .4000834 .3152819 .6665 865.1642974 .2767378 .0016741 .8255618 .5433901 .0897 904.9481675 .9644948 .4349968 .0743481 .2220384 .9168

The proposed algorithm is compared with the works on Hadamard transfrom already present in literature Ref.[28], and Ref.[6]. The peak signal to noise ratio of Ref.[6] is around $45 \mathrm{~dB}$. The Peak signal to noise ratio of proposed algorithm is greater than $54 \mathrm{~dB}$. References Ref. [6] and Ref.[28] are used for comparing the proposed algorithm in Table. 5 and Table 3 and the high N.C.C value of proposed algorithm indicate that it is more robust to attacks.

The host and watermarked images are shown in Figure. 7 and 8 The various attacks used in watermarking are

(1) Sharpening: The watermarked image is subjected to $3 \times 3$ mask of high pass filter. The extracted watermark is $95 \%$ similar to original watermark.

(2) averaging: The watermarked image is subjected to $3 \times 3$ mask of low pass filter. The extracted watermark is $97 \%$ is similar to original watermark.

(3) Rotation is one of the major attack in image watermarking.Normally when image is rotated the size of image is in- 


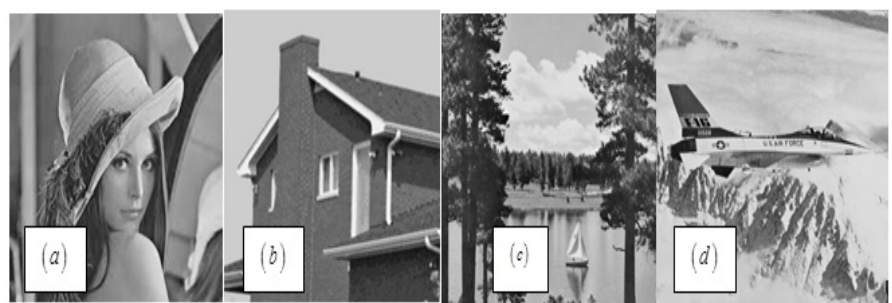

Fig. 7. Host images

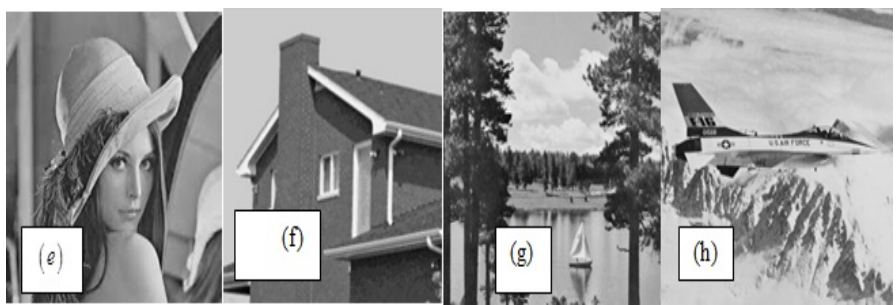

Fig. 8. Watermarked images

creased. so in order to restore original shape some of the useful information is lost. The retrieved watermark is $95 \%$ similar to original watermark.

(4) Scaling is a major attack in image watermarking. It removes high frequency components. So useful information may be lost. In the proposed watermarking the image is scaled to one fourth of the original and restored back to its normal size before extraction. The retrieved watermark is $98 \%$ similar to original watermark.

(5) Gaussian noise : Gaussian noise with zero mean and normalized variance 0.01 are added to distorted image.The retrieved watermark is $95 \%$ similar to original watermark.

(6) Salt and pepper noise: It adds this noise to the watermarked image, with specified noise density. The default value of noise density taken is 0.05 .The retrieved watermark is $97 \%$ similar to original watermark.

(7) Motion blur: Apply motion blur to the watermarked image. The retrieved watermark is $90 \%$ similar to original watermark.

(8) Circular blur: Apply circular blur to watermarked image.The retrieved watermark is $95 \%$ similar to original watermark.

(9) Vertical flip: It returns watermarked image with rows flipped in the up-down direction, that is, about a horizontal axis.The retrieved watermark is $98 \%$ similar to original watermark.

(10) Horizontal flip: It returns watermarked image with columns flipped in the left-right direction, that is, about a vertical axis.

(11) JPEG compression: It compresses the watermarked image to the specified jpeg quality factor of $60 \%$.The retrieved watermark is $98 \%$ similar to original watermark.

(12) Cropping: It crops the watermarked image in specified location up to a height and width.The retrieved watermark is $98 \%$ similar to original watermark.

The algorithm is trained with different host images -Lena, Boat, Jet plane and House as shown in Figure. 7 with G.R.I.E.T logo. Each image have different histograms. Though the images are different with the proposed scaling factors obtained with GA using the
Table 5. Comparision of Normalized cross correlation between original and extracted watermark for different cover images in proposed algorithm

\begin{tabular}{ccccc}
\hline \hline Attack type & Lena & House & Jetplane & Boat \\
\hline \hline Sharpening & 0.9724 & 0.9889 & 0.9183 & 0.9743 \\
Averaging & 0.9771 & 0.9876 & 0.9523 & 0.9772 \\
Scaling & 0.9777 & 0.9458 & 0.9558 & 0.9779 \\
Rotation & 0.9448 & 0.9458 & 0.9558 & 0.9420 \\
Salt and Pepper noise & 0.9492 & 0.9679 & 0.9288 & 0.9469 \\
Gaussian noise & 0.9607 & 0.9776 & 0.9383 & 0.9597 \\
Motion Blur & 0.9508 & 0.9445 & 0.9140 & 0.9505 \\
Disk Blur & 0.9302 & 0.9053 & 0.8675 & 0.9300 \\
Horizontal flip & 0.9777 & 0.9870 & 0.9607 & 0.9799 \\
Vertical flip & 0.9777 & 0.9870 & 0.9607 & 0.9799 \\
Cropping & 0.9777 & 0.9850 & 0.9752 & 0.9813 \\
Jpeg Compression & 0.9803 & 0.9875 & 0.9756 & 0.9779 \\
\hline
\end{tabular}

trained host images the N.C.C is found to be more than $95 \%$. The corresponding watermarked images are shown in Fig. 8

\section{CONCLUSION}

Inherently Walsh-Hadamard transform is suffered with the problem of robustness. In watermarking optimizing transparency and robustness is a challenge. By using multiple scaling factors derived from GA, an amelioration of transparency and robustness is achieved in the proposed algorithm.But GA suffers from high computational complexity. So in future the work can be extended with Differential Evolution that has the advantages of fast convergence,minimum parameters to control and parallel processing.

\section{REFERENCES}

[1] Emad E Abdallah, A Ben Hamza, and Prabir Bhattacharya. Mpeg video watermarking using tensor singular value decomposition. In Image Analysis and Recognition, pages 772-783. Springer, 2007.

[2] Charu Agarwal, Anurag Mishra, and Arpita Sharma. Grayscale image watermarking using gabpn hybrid network. Journal of Visual Communication and Image Representation, 24(7):1135-1146, 2013.

[3] Harry C Andrews and C Patterson III. Singular value decomposition (svd) image coding. Communications, IEEE Transactions on, 24(4):425-432, 1976.

[4] Veysel Aslantas. A singular value decomposition-based image watermarking using genetic algorithm. $A E U$ International Journal of Electronics and Communications, 62(5):386-394, 2008.

[5] Veysel Aslantas. An optimal robust digital image watermarking based on svd using differential evolution algorithm. $\mathrm{Op}$ tics Communications, 282(5):769-777, 2009.

[6] Gaurav Bhatnagar and Balasubramanian Raman. Robust watermarking in multiresolution walsh-hadamard transform. In Advance Computing Conference, 2009. IACC 2009. IEEE International, pages 894-899. IEEE, 2009.

[7] Alexia Briassouli and Michael G Strintzis. Locally optimum nonlinearities for dct watermark detection. Image Processing, IEEE Transactions on, 13(12):1604-1617, 2004.

[8] Wen-Yuan Chen and Chin-Hsing Chen. A robust watermarking scheme using phase shift keying with the combination of 
amplitude boost and low amplitude block selection. Pattern Recognition, 38(4):587-598, 2005.

[9] BJ Falkowski and Lip-San Lim. Image watermarking using hadamard transforms. Electronics Letters, 36(3):211-213, 2000 .

[10] BJ Falkowski and S Rahardja. Walsh-like functions and their relations. In Vision, Image and Signal Processing, IEE Proceedings-, volume 143, pages 279-284. IET, 1996.

[11] Bogdan J Falkowski. Properties and ways of calculation of multi-polarity generalized walsh transforms. Circuits and Systems II: Analog and Digital Signal Processing, IEEE Transactions on, 41(6):380-391, 1994.

[12] Emir Ganic and Ahmet M Eskicioglu. Robust embedding of visual watermarks using discrete wavelet transform and singular value decomposition. Journal of Electronic Imaging, 14(4):043004-043004, 2005.

[13] Rafael C Gonzalez and E Richard. Woods, digital image processing. ed: Prentice Hall Press, ISBN 0-201-18075-8, 2002.

[14] Prayoth Kumsawat, Kitti Attakitmongcol, and Arthit Srikaew. A new approach for optimization in image watermarking by using genetic algorithms. Signal Processing, IEEE Transactions on, 53(12):4707-4719, 2005.

[15] Martin Kutter, Sviatoslav V Voloshynovskiy, and Alexander Herrigel. Watermark copy attack. In Electronic Imaging, pages 371-380. International Society for Optics and Photonics, 2000.

[16] Shinfeng D Lin, Shih-Chieh Shie, and Jim Yi Guo. Improving the robustness of dct-based image watermarking against jpeg compression. Computer Standards \& Interfaces, 32(1):5460, 2010.

[17] Jian-Chyn Liu and Shu-Yuan Chen. Fast two-layer image watermarking without referring to the original image and watermark. Image and Vision Computing, 19(14):1083-1097, 2001.

[18] Ruizhen Liu and Tieniu Tan. An svd-based watermarking scheme for protecting rightful ownership. Multimedia, IEEE Transactions on, 4(1):121-128, 2002.

[19] Yan Liu and Jiying Zhao. A new video watermarking algorithm based on $1 \mathrm{~d} \mathrm{dft}$ and radon transform. Signal Processing, 90(2):626-639, 2010.

[20] Santi P Maity and Claude Delpha. Optimization in digital watermarking techniques. Advanced Techniques in Multimedia Watermarking: Image, Video and Audio Application, IGI Global Pub., USA, pages 369-406, 2010.

[21] K Meenakshi, Ch Srinivasa Rao, and K Satya Prasad. A fast and robust hybrid watermaking scheme based on schur and svd transform. International Journal of Research in Engineering and Technology, 3(4):7-11, 2014.

[22] K Meenakshi, Ch Srinivasa Rao, K Satya Prasad, et al. Robust video watermarking schemes in phase domain using binary phase shift keying. arXiv preprint arXiv:1404.1314, 2014.

[23] K Meenakshi, Ch Srinivasa Rao, and K Satya Prasad. A scene based video watermarking using slant transform. IETE Journal of Research, (ahead-of-print):1-12, 2014.

[24] Jagdish C Patra, Jiliang E Phua, and Cedric Bornand. A novel dct domain crt-based watermarking scheme for image authentication surviving jpeg compression. Digital Signal Processing, 20(6):1597-1611, 2010.
[25] William Pratt, Julius Kane, and Harry C Andrews. Hadamard transform image coding. Proceedings of the IEEE, 57(1):5868,1969

[26] Mahalingam Ramkumar and Ali N Akansu. Capacity estimates for data hiding in compressed images. Image Processing, IEEE Transactions on, 10(8):1252-1263, 2001.

[27] JJK Ó Ruanaidh, WJ Dowling, and FM Boland. Watermarking digital images for copyright protection. IEE ProceedingsVision, Image and Signal Processing, 143(4):250-256, 1996.

[28] V Santhi and P Arulmozhivarman. Hadamard transform based adaptive visible/invisible watermarking scheme for digital images. Information Security Technical Report, 2013.

[29] Adrian Sequeira and Deepa Kundur. Communication and information theory in watermarking: A survey. In ITCom 2001: International Symposium on the Convergence of IT and Communications, pages 216-227. International Society for Optics and Photonics, 2001.

[30] Chin-Shiuh Shieh, Hsiang-Cheh Huang, Feng-Hsing Wang, and Jeng-Shyang Pan. Genetic watermarking based on transform-domain techniques. Pattern recognition, 37(3):555-565, 2004

[31] Jieh-Ming Shieh, Der-Chyuan Lou, and Ming-Chang Chang. A semi-blind digital watermarking scheme based on singular value decomposition. Computer Standards \& Interfaces 28(4):428-440, 2006.

[32] Zhou Wang, Alan C Bovik, Hamid R Sheikh, and Eero P Simoncelli. Image quality assessment: from error visibility to structural similarity. Image Processing, IEEE Transactions on, 13(4):600-612, 2004.

[33] Iran Yazd. Digital image watermarking based on parameters amelioration of parametric slant-hadamard transform using genetic algorithm. 2012

[34] Dong Zheng, Jiying Zhao, and Abdulmotaleb El Saddik. Rstinvariant digital image watermarking based on log-polar mapping and phase correlation. Circuits and Systems for Video Technology, IEEE Transactions on, 13(8):753-765, 2003. 